Rainer Scheid

Raymond Voltz

Thomas Vetter

Osama Sabri

D. Yves von Cramon

\section{Neurosyphilis and paraneoplastic limbic encephalitis: important differential diagnoses}

Received: 14 September 2004

Received in revised form: 4 January 2005

Accepted: 27 January 2005

Published online: 1 April 2005

Sirs: A 34-year-old previously healthy heterosexual man with a history of alcohol and tobacco abuse was admitted to hospital after a first epileptic seizure. Neurological examination was unremarkable. Lumbar puncture was performed the same day with the following results: 4 cells $/ \mathrm{mm}^{3}$, protein $953 \mathrm{mg} / \mathrm{l}$. Non-contrast CT was normal. EEG showed generalized slowing and epileptiform discharges. At first, symptomatic epilepsy in the context of alcoholism was suspected. During the following days, repeated complex partial seizures developed. The patient was disoriented, anterograde and retrograde memory were severely impaired. On the $4^{\text {th }}$ day, MRI showed a contrast-enhancing (T1-) hyperintense signal alteration in the left medial temporal lobe on FLAIR and T2-weighted images (Fig. 1a). Repeat CSF-analysis showed 22 cells $/ \mathrm{mm}^{3}$, protein $952 \mathrm{mg} / \mathrm{l}$, and positive oligoclonal bands. Serum/CSF tests for HSV $1 / 2$, VZV, HIV $1 / 2$ were negative. HHV-6-Ab titer in serum was 1:20 (IgG). VDRL in serum was positive, titer 1:8, in CSF 1:4. Quantitative TPPA-test in serum was 1:81920, in CSF 1:524288. Treponema pallidum IgG-Western Blot was positive in serum and CSF. Neurosyphilis (NSP) was diagnosed and IV penicillin treatment at a dose of $3 \times 10$ million units/d for 20 days was initiated. (Note: US Centers for Disease Control and Prevention recommended treatment for NSP [18]: penicillin G 18-24 million units/d administered as 3-4 million units IV every 4 hours or continuous infusion for 14 days).

Because of the MRI findings suggestive of limbic encephalitis [7, 12], paraneoplastic limbic encephalitis (PLE) was considered as a differential diagnosis. Anti-neuronal antibodies (anti-Amphiphysin, -Hu, - Ri, -CV2, -Ma2) were negative. Whole body F-18FDG-PET showed a focal hypometabolism in the left medial temporal lobe and a circumscribed area of increased tracer uptake in the left upper lung (Fig. 1b). Thoracic CT correspondingly showed a hyperdense lesion of $1.6 \mathrm{~cm}$ in the left superior pulmonary lobe, suspicious of carcinoma. Wedge resection of the left superior pulmonary lobe was performed. Histopathological analysis showed a demarcated severe chronic inflammation with fibrosis (non-caseous degeneration). Treponema pallidum PCR was negative. There were no signs of malignancy.

Under continuous anticonvulsant therapy no more seizures occurred. The patient's state subsequently improved. However, after 8 months of follow up the patient still suffered from generalized cognitive slowing, impaired anterograde memory- and executive functions. Repeat MRI showed atrophy of left medial temporal lobe structures.

Although NSP has become rare in industrialized countries [9], it has to be considered in a variety of neuropsychiatric disorders. PLE is another infrequent CNS disorder, which manifests itself in the presence of an - initially frequently occult - malignancy. Patients with
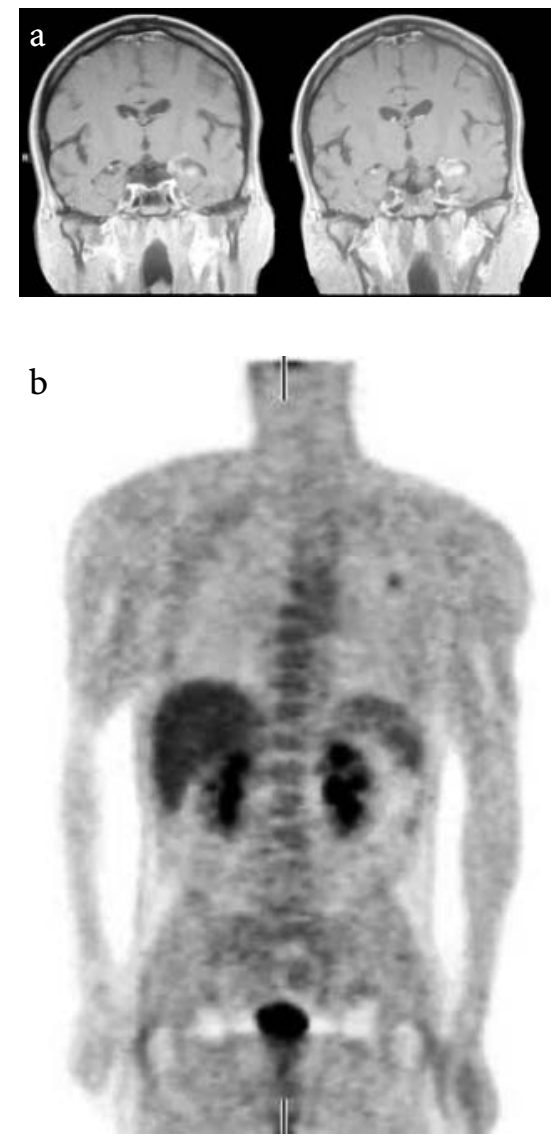

Fig. 1 a T1-weighted MRI in the coronal plane showing a contrast enhancing lesion in the left hippocampal formation. b Whole-body FDG-PET scan; coronal plane. Circumscribed, pathologically increased glucose metabolism in the left superior pulmonary lobe. Correspondingly, thoracic CT showed a hyperdense lesion of $1.6 \mathrm{~cm}$, suspicious of a peripheral lung carcinoma (not shown)

PLE can harbor anti-neuronal antibodies, most often anti-Hu or antiMa2, which, however, are not obligatory [11]. Because of their unspecific or atypical clinical course, the spectrum of differential diagnoses of both diseases is wide. Particularly NSP has always been considered "the great imitator". Nevertheless, to our knowledge these entities have never been reported as direct differential diagnoses.

Clinical hallmarks of PLE are memory dysfunction, epilepsy, and psychiatric abnormalities [17]. The 
same is true for our patient and certain previously reported patients with NSP. However, predominant medial temporal lobe involve- ment seems to be rare in NSP, since there are only anecdotal case reports of this particular variant in the MEDLINE database $[1-6,8,14$,
15, 19-21, 23] (Table 1). Furthermore, since reversible temporal lobe MRI abnormalities have been reported also in the context of par-

Table 1 Overview of the previously published patients with NSP and isolated or prominent medial temporal lobe involvement demonstrating a close similarity of clinical, neuroimaging, and laboratory findings as compared to PLE (CE contrast enhancement; ea epileptic activity; gs generalized slowing; / left; $m v$ meningovascular; $n d$ not done or no data available; neg negative; $p a$ parenchymal; pos positive; $r$ right; $W M$ white matter)

\begin{tabular}{|c|c|c|c|c|c|c|c|c|c|c|c|c|c|}
\hline Author & $\begin{array}{l}\text { Number, } \\
\text { age, and } \\
\text { sex of } \\
\text { patients }\end{array}$ & $\begin{array}{l}\text { Symptoms } \\
\text { and signs }\end{array}$ & $\begin{array}{l}\text { Acuity of } \\
\text { symptoms }\end{array}$ & $\begin{array}{l}\text { MRI - } \\
\text { Sequence } \\
\text { and side } \\
\text { of medial } \\
\text { temporal } \\
\text { findings }\end{array}$ & $\begin{array}{l}\text { MRI - } \\
\text { CE }\end{array}$ & $\begin{array}{l}\text { MRI- } \\
\text { Additional } \\
\text { lesions }\end{array}$ & $\begin{array}{l}\text { MRI - } \\
\text { follow-up }\end{array}$ & EEG & $\begin{array}{l}\text { CSF- } \\
\text { protein } \\
{[\mathrm{g} / \mathrm{l}]}\end{array}$ & $\begin{array}{l}\text { CSF- } \\
\text { cells } \\
{\left[1 / \mathrm{mm}^{3}\right]}\end{array}$ & $\begin{array}{l}\text { Serum } \\
\text { VDRL } \\
\text { RPR } \\
\text { [titer] }\end{array}$ & $\begin{array}{l}\text { CSF } \\
\text { VDRL } \\
\text { [titer] }\end{array}$ & $\begin{array}{l}\text { Type } \\
\text { of } \\
\text { NSP }\end{array}$ \\
\hline $\begin{array}{l}\text { Ances } \\
\text { et al. [1] }\end{array}$ & $1 ; 41, \mathrm{~m}$ & $\begin{array}{l}\text { memory } \\
\text { impairment, } \\
\text { epilepsy }\end{array}$ & acute & $\mathrm{I}^{\mathrm{T}}$ & nd & $\begin{array}{l}\text { lateral } \\
\text { temporal lobe } \\
\text { I }\end{array}$ & nd & ea & 1.17 & 43 & $1: 32$ & $1: 16$ & nd \\
\hline $\begin{array}{l}\text { Angus } \\
\text { et al. [2] }\end{array}$ & $1 ; 34, \mathrm{~m}$ & $\begin{array}{l}\text { disorientation, } \\
\text { agitation, } \\
\text { aphasia }\end{array}$ & $\begin{array}{l}\text { subacute } \\
\text { (3 days) }\end{array}$ & $\mathrm{T} 1$ & yes & $\begin{array}{l}\text { leptomeningeal } \\
\text { enhancement }\end{array}$ & nd & ea & 0.94 & 26 & $1: 32$ & $1: 2$ & $\mathrm{mv}$ \\
\hline $\begin{array}{l}\text { Bash } \\
\text { et al. [3] }\end{array}$ & $1 ; 50, \mathrm{~m}$ & $\begin{array}{l}\text { disorientation, } \\
\text { memory } \\
\text { impairment, } \\
\text { epilepsy }\end{array}$ & $\begin{array}{l}\text { subacute } \\
\text { (3 months) }\end{array}$ & $\begin{array}{l}T 2 \\
\text { FLAIR } \\
r>I\end{array}$ & no & none & $\begin{array}{l}\text { regression of } \\
\text { hyperintensities, } \\
\text { atrophy of temporal } \\
\text { lobe structures r } \\
\text { ( } 4 \text { months) }\end{array}$ & gs & 0.87 & 19 & $1: 64$ & $1: 16$ & $\mathrm{mv}$ \\
\hline $\begin{array}{l}\text { Berbel- } \\
\text { Garcia } \\
\text { et al. [4] }\end{array}$ & $1 ; 47, \mathrm{~m}$ & $\begin{array}{l}\text { disorientation, } \\
\text { memory } \\
\text { impairment, } \\
\text { personality } \\
\text { changes }\end{array}$ & $\begin{array}{l}\text { slowly } \\
\text { progressive } \\
\text { (24 months) }\end{array}$ & $\mathrm{T} 2$ & nd & $\begin{array}{l}\text { lateral } \\
\text { temporal lobe, } \\
\text { frontal lobe } \\
r\end{array}$ & $\begin{array}{l}\text { regression of } \\
\text { hyperintensities }\end{array}$ & nd & 0.64 & 5 & $1: 64$ & $1: 64$ & pa \\
\hline $\begin{array}{l}\text { Blanco } \\
\text { et al. [5] }\end{array}$ & $1 ; 46, \mathrm{~m}$ & $\begin{array}{l}\text { apathy, } \\
\text { memory } \\
\text { impairment }\end{array}$ & $\begin{array}{l}\text { slowly } \\
\text { progressive } \\
\text { (12 months) }\end{array}$ & $\begin{array}{l}T 2 \\
I=r\end{array}$ & nd & $\begin{array}{l}\text { temporal pole, } \\
\text { insula, } \\
\text { subcortical } \\
\text { WM } \\
\text { I=r }\end{array}$ & normalization & gs & 1.18 & 140 & $1: 16$ & neg & $\mathrm{mv}$ \\
\hline $\begin{array}{l}\text { Denays } \\
\text { et al. [6] }\end{array}$ & $1 ; 51, f$ & $\begin{array}{l}\text { disorientation, } \\
\text { memory } \\
\text { impairment }\end{array}$ & acute & $\begin{array}{l}\text { FLAIR } \\
I>r\end{array}$ & nd & none & nd & ea & 0.46 & 23 & nd & nd & pa \\
\hline $\begin{array}{l}\text { Fujimoto } \\
\text { et al. [8] }\end{array}$ & $1 ; 41, \mathrm{~m}$ & $\begin{array}{l}\text { disorientation, } \\
\text { memory } \\
\text { impairment }\end{array}$ & $\begin{array}{l}\text { subacute } \\
\text { ( } 4 \text { months) }\end{array}$ & $\begin{array}{l}T 2, \\
D P \\
I=r\end{array}$ & nd & $\begin{array}{l}\text { caudate } \\
\text { nucleus I, } \\
\text { thalamus r }\end{array}$ & $\begin{array}{l}\text { regression of } \\
\text { hyperintensities } \\
\text { (1 month) }\end{array}$ & nd & 1.45 & 122 & $1: 16$ & nd & pa \\
\hline $\begin{array}{l}\text { Lauria } \\
\text { et al. [14] }\end{array}$ & $1 ; 62, \mathrm{~m}$ & $\begin{array}{l}\text { aphasia, } \\
\text { hemiparesis, } \\
\text { epilepsy }\end{array}$ & acute & $\begin{array}{l}\text { FLAIR } \\
r\end{array}$ & yes & $\begin{array}{l}\text { caudate } \\
\text { nucleus, } \\
\text { gyrus cinguli, } \\
\text { inferior } \\
\text { frontal lobe r }\end{array}$ & $\begin{array}{l}\text { atrophy of temporal } \\
\text { lobe structures } r \\
\text { ( } 4 \text { months) }\end{array}$ & nd & nd & 5 & pos & pos & pa \\
\hline $\begin{array}{l}\text { Marano } \\
\text { et al. [15] }\end{array}$ & $1 ; 48, \mathrm{~m}$ & $\begin{array}{l}\text { memory } \\
\text { impairment, } \\
\text { epilepsy }\end{array}$ & acute & $\begin{array}{l}\text { T2, } \\
\text { DP } \\
r\end{array}$ & no & $\begin{array}{l}\text { basal frontal } \\
\text { lobe } \\
r\end{array}$ & $\begin{array}{l}\text { regression of } \\
\text { hyperintensities, } \\
\text { atrophy of temporal } \\
\text { lobe structures r } \\
\text { (1 month) }\end{array}$ & nd & 0.63 & 12 & pos & pos & $\mathrm{mv}$ \\
\hline $\begin{array}{l}\text { Scheid } \\
\text { et al. } \\
\text { (current } \\
\text { report) }\end{array}$ & $1 ; 34, \mathrm{~m}$ & $\begin{array}{l}\text { disorientation, } \\
\text { memory } \\
\text { impairment, } \\
\text { epilepsy }\end{array}$ & acute & $\begin{array}{l}\text { T2, } \\
\text { FLAIR } \\
\text { I }\end{array}$ & yes & none & $\begin{array}{l}\text { atrophy of medial } \\
\text { temporal lobe } \\
\text { structures I } \\
\text { (4 months) }\end{array}$ & $\begin{array}{l}\text { ea } \\
\text { gs }\end{array}$ & 0.95 & 4 & $1: 8$ & $1: 4$ & pa \\
\hline $\begin{array}{l}\text { Silber- } \\
\text { stein } \\
\text { et al. [19] }\end{array}$ & $1 ; 37, \mathrm{~m}$ & $\begin{array}{l}\text { cognitive } \\
\text { decline, } \\
\text { memory } \\
\text { impairment, } \\
\text { deafness, } \\
\text { epilepsy }\end{array}$ & $\begin{array}{l}\text { slowly } \\
\text { progressive } \\
\text { (24 months) }\end{array}$ & $\begin{array}{l}\text { FLAIR } \\
I>r\end{array}$ & no & $\begin{array}{l}\text { frontal lobe, } \\
\text { insula } \\
I>r\end{array}$ & nd & gs & 1.43 & 32 & $1: 128$ & 1:128 & pa \\
\hline
\end{tabular}


Table 1 Continued

\begin{tabular}{|c|c|c|c|c|c|c|c|c|c|c|c|c|c|}
\hline Author & $\begin{array}{l}\text { Number, } \\
\text { age, and } \\
\text { sex of } \\
\text { patients }\end{array}$ & $\begin{array}{l}\text { Symptoms } \\
\text { and signs }\end{array}$ & $\begin{array}{l}\text { Acuity of } \\
\text { symptoms }\end{array}$ & $\begin{array}{l}\text { MRI - } \\
\text { Sequence } \\
\text { and side } \\
\text { of medial } \\
\text { temporal } \\
\text { findings }\end{array}$ & $\begin{array}{l}\text { MRI - } \\
\text { CE }\end{array}$ & $\begin{array}{l}\text { MRI - } \\
\text { Additional } \\
\text { lesions }\end{array}$ & $\begin{array}{l}\text { MRI - } \\
\text { follow-up }\end{array}$ & EEG & $\begin{array}{l}\text { CSF- } \\
\text { protein } \\
{[\mathrm{g} / \mathrm{l}]}\end{array}$ & $\begin{array}{l}\text { CSF- } \\
\text { cells } \\
{\left[1 / \mathrm{mm}^{3}\right]}\end{array}$ & $\begin{array}{l}\text { Serum } \\
\text { VDRL } \\
\text { RPR } \\
\text { [titer] }\end{array}$ & $\begin{array}{l}\text { CSF } \\
\text { VDRL } \\
\text { [titer] }\end{array}$ & $\begin{array}{l}\text { Type } \\
\text { of } \\
\text { NSP }\end{array}$ \\
\hline $\begin{array}{l}\text { Szilak } \\
\text { et al. [20] }\end{array}$ & $1 ; 55, \mathrm{~m}$ & $\begin{array}{l}\text { disorientation, } \\
\text { confusion, } \\
\text { memory } \\
\text { impairment, } \\
\text { epilepsy }\end{array}$ & acute & $\begin{array}{l}\text { T2, } \\
\text { FLAIR } \\
\text { I }\end{array}$ & nd & $\begin{array}{l}\text { subcortical } \\
\text { WM }\end{array}$ & nd & ea & 0.71 & 79 & $1: 64$ & $1: 8$ & $\mathrm{mv}$ \\
\hline $\begin{array}{l}\text { Vojvodic } \\
\text { et al. [21] }\end{array}$ & $1 ; 45, \mathrm{~m}$ & $\begin{array}{l}\text { mental } \\
\text { confusion, } \\
\text { memory } \\
\text { impairment, } \\
\text { epilepsy }\end{array}$ & $\begin{array}{l}\text { slowly } \\
\text { progressive } \\
\text { (14 months) }\end{array}$ & $\begin{array}{l}T 2 \\
I=r\end{array}$ & nd & none & nd & nd & 0.53 & 9 & $1: 32$ & nd & nd \\
\hline $\begin{array}{l}\text { Zifko } \\
\text { et al. [23] }\end{array}$ & $1 ; 35, \mathrm{~m}$ & $\begin{array}{l}\text { memory } \\
\text { impairment, } \\
\text { depression }\end{array}$ & $\begin{array}{l}\text { slowly } \\
\text { progressive } \\
\text { ( } 2 \text { years) }\end{array}$ & $\begin{array}{l}\text { FLAIR } \\
I=r\end{array}$ & nd & $\begin{array}{l}\text { hippocampal } \\
\text { and frontal } \\
\text { atrophy }\end{array}$ & $\begin{array}{l}\text { unchanged } \\
\text { (12 months) }\end{array}$ & nd & nd & nd & neg & $1: 2$ & pa \\
\hline
\end{tabular}

tial status epilepticus [13], epilepsy has to be discussed as a confounder in the assessment of medial temporal lobe abnormalities in NSP, at least in the 10 patients of Table 1 with clinical or EEG evidence of epilepsy. In addition, it is interesting to note that the majority of these patients $(8 / 10)$ had an acute or subacute onset of symptoms, whereas 3 of the remaining 4 patients without seizures had a slowly progressive course of the disease. It therefore could be speculated that the etiology of medial temporal lobe abnormalities might differ in NSP with and without epilepsy.

The pathogeneses of the MRI findings are unknown, both in PLE and in the reported variant of NSP. In the latter, several authors regard them as meningovascular $[2,3,5$, $15,20]$. However, the imaging abnormalities do not reflect a vascular territory and neuropathologically, arteritis is usually seen in large and medium-sized blood vessels in NSP [10]. The hypothesis of parenchymal syphilis is thus more convincing. Contrast enhancement may be observed in both disorders and is no criterion of differentiation (Table 1).

It is noteworthy that despite the frequent association of AIDS and syphilis, neither our not any of the previously reported patients has been tested HIV-positive. In analogy to the assumed autoimmune origin of PLE, this observation may indicate that a competent immune response is a prerequisite for the development of non-tumorous medial temporal lobe damage.

This case report demonstrates the possible complexity of the differential diagnostic procedure. FDG-PET has been reported to be useful in the diagnosis of paraneoplastic neurological syndromes [16]. However, it must be emphasized that FDG-PET findings are unspecific and might lead to wrong conclusions. Our observation therefore supports the conclusion of a current study that FDG-PET results should be interpreted with caution in patients without antineuronal antibodies [22].

In conclusion, neurosyphilis has to be excluded if paraneoplastic limbic encephalitis is suspected, and vice versa.

Acknowledgements We thank Professor J. Honnorat, Lyon, France for his critical review of the manuscript. We also thank Professor H. J. Hagedorn, Herford, Germany for having performed Treponema pallidum PCR out of probes of the lung resection of the patient.

\section{References}

1. Ances BM, Shellhaus R, Brown MJ, Rios OV, Herman ST, French JA (2004) Neurosyphilis and status epilepticus: case report and literature review. Epilepsy Res 59:67-70

2. Angus F, Maysuria H, Bryan CS (1998) Neurosyphilis mimicking herpes simplex encephalitis. J S C Med Assoc 94: 315-317

3. Bash S, Hathout GM, Cohen S (2001) Mesiotemporal T2-weighted hyperintensity: neurosyphilis mimicking herpes encephalitis. AJNR Am J Neuroradiol 22:314-316

4. Berbel-Garcia A, Porta-Etessam J, Martinez-Salio A, Millan-Juncos JM, PerezMartinez DA, Saiz-Diaz RA, ToledoHeras M (2004) Magnetic resonance image-reversible findings in a patient with general paresis. Sex Transm Dis 31:350-352

5. Blanco Y, Sánchez-Valle R, Valldeoriola F (2002) Bilateral temporal leukoencephalopathy in neurosyphilis. [Spanish] Neurología 17:52

6. Denays R, Collier A, Rubinstein M, Atsama PA (1999) 51-year-old woman with disorientation and amnesia. Lancet 354:1786

7. Dirr LY, Elster AD, Donofrio PD, Smith M (1990) Evolution of brain MRI abnormalities in limbic encephalitis. Neurology 40:1304-1306

8. Fujimoto $H$, Imaizumi $T$, Nishimura $Y$, Miura Y, Ayabe M, Shoji H, Abe T (2001) Neurosyphilis showing transient global amnesia-like attacks and magnetic resonance imaging abnormalities mainly in the limbic system. Intern Med 40:439-442 
9. Golden MR, Marra CM, Holmes KK (2003) Update on syphilis. Resurgence of an old problem. JAMA 290: 1510-1514

10. Gray F, Alonso JM (2002) Spirochaetal infections. In: Graham DI, Lantos PL (eds) Greenfield's Neuropathology. $7^{\text {th }}$ ed. London, New York: Arnold, pp 138-144

11. Gultekin SH, Rosenfeld MR, Voltz R, Eichen J, Posner JB, Dalmau J (2000) Paraneoplastic limbic encephalitis: neurological symptoms, immunological findings and tumour association in 50 patients. Brain 123:1481-1494

12. Kalkman PH, Allan S, Birchall IAWJ (1993) Magnetic resonance imaging of limbic encephalitis. Can Assoc Radiol J 44:121-124

13. Kavuk I, Koeppen S, Agelink M, Dörfler A, Limmroth V, Diener HC (2004) Transient MRI abnormalities associated with partial status epilepticus. J Neurol 251:1156-1157

14. Lauria G, Erbetta A, Pareyson D, Sghirlanzoni A (2001) Parenchymatous neurosyphilis. Neurol Sci 22:281-282

15. Marano E, Briganti F, Tortora F, Elefante A, De Rosa A, Maiuri F, Filla A (2004) Neurosyphilis with complex partial status epilepticus and mesiotemporal MRI abnormalities mimicking herpes simplex encephalitis. J Neurol Neurosurg Psychiatry $75: 833$
16. Rees JH, Hain SF, Johnson MR, Hughes RAC, Costa DC, Ell PJ, Keir G, Rudge P (2001) The role of $\left[{ }^{18} \mathrm{~F}\right]$ fluoro-2-deoxyglucose-PET scanning in the diagnosis of paraneoplastic neurological disorders. Brain 124:2223-2231

17. Scheid R, Voltz R, Guthke T, Sammler D, von Cramon DY (2003) Neuropsychiatric findings in anti-Ma2-positive paraneoplastic limbic encephalitis. Neurology 61:1159-1161

18. Sexually transmitted diseases treatment guidelines (2002) MMWR Recomm Rep 51:1-78

19. Silberstein P, Lawrence R, Pryor D, Shnier RA (2002) case of neurosyphilis with a florid Jarisch-Herxheimer reaction. J Clin Neurosci 9:689-690

20. Szilak I, Marty F, Helft J, Soeiro R (2001) Neurosyphilis presenting as herpes simplex encephalitis. Clin Infect Dis 32:1108-1109

21. Vojvodic NM, Sokic DV, Jankovic SM, Delic S (2003) Isolated episodes of status epilepticus as the manifestation of neurosyphilis: a case report. Epilepsia 44:623

22. Younes-Mhenni S, Janier MF, Cinnoti L, Antoine JC, Tronc F, Cottin V, Ternamian PJ, Trouillas P, Honnorat J (2004) 18FDG-PET improves tumor detection in patients with paraneoplastic neurological syndromes. Brain 127:2331-2338
23. Zifko U, Wimberger D, Lindner $\mathrm{K}$, Zier G, Grisold W, Schindler E (1996) MRI in patients with general paresis. Neuroradiology $38: 120-123$

Dr. R. Scheid (两) ·

D. Y. von Cramon, $\mathrm{MD}, \mathrm{PhD}$

Day Clinic of Cognitive Neurology

University of Leipzig

Liebigstr. 22a

04103 Leipzig, Germany

Tel.: + 49-341/9724-980

Fax: + 49-341/9724-989

E-Mail: scheid@cbs.mpg.de

R. Scheid, MD • D. Y. von Cramon, MD, PhD Max Planck Institute for Human Cognitive and Brain Sciences

Leipzig, Germany

R. Voltz, MD, PhD

Dept. of Palliative Medicine

University of Cologne

Cologne, Germany

T. Vetter, MD

Sächsisches Krankenhaus Altscherbitz

Dept. of Neurology

Schkeuditz, Germany

O. Sabri, MD, PhD

Dept. of Nuclear Medicine

University of Leipzig

Leipzig, Germany 